\title{
CARACTERIZAÇÃO MORFOLÓGICA DE ACESSOS DE GERMOPLASMA DE QUATRO ESPÉCIES BRASILEIRAS DE AMENDOIM-SILVESTRE ${ }^{(1)}$
}

\author{
RENATO FERRAZ DE ARRUDA VEIGA ${ }^{(2,5)}$; RACHEL BENETTI \\ QUEIROZ-VOLTAN $^{(2)}$; JOSÉ FRANCISCO MONTENEGRO VALLS ${ }^{(3)}$; \\ ALESSANDRA PEREIRA FÁVERO ${ }^{(4)}$; WILSON BARBOSA ${ }^{(2,5)}$ \\ RESUMO
}

\begin{abstract}
Caracterizaram-se morfologicamente os acessos de germoplasma de espécies silvestres brasileiras de amendoim do gênero Arachis L., Sect. Arachis e analisaram-se a similaridade genética entre acessos da mesma espécie e entre as espécies. Realizou-se o experimento nos anos agrícolas de 1993 a 1996, no Núcleo Experimental de Campinas, do Instituto Agronômico (IAC). Avaliaram-se os acessos disponíveis no Banco Ativo de Germoplasma de Espécies Silvestres de Arachis, da Embrapa Recursos Genéticos e Biotecnologia (CENARGEN - Brasília, DF), das espécies A. palustris Krapov., W.C. Gregory \& Valls, A. decora Krapov., W.C. Gregory \& Valls, A. praecox Krapov., W.C. Gregory \& Valls e A. stenosperma Krapov. \& W.C. Gregory, efetuando-se anotações fenotípicas quantitativas e qualitativas, conforme lista de descritores morfológicos. Observou-se que os acessos de A. stenosperma são semelhantes, apesar da sua grande distância geográfica, e diferem das demais espécies, formando um grupo mais coeso. Caracteres como o diâmetro do eixo central e o comprimento dos frutos e das sementes serviram para distingui-la das demais espécies. Arachis decora apresentou alta variação entre acessos nos vários caracteres morfológicos estudados. A. palustris apresentou alta variação morfológica entre acessos, ainda que tenham sido analisados apenas dois, para altura da planta, largura da semente, dimensões do esporão, istmo, folíolo, raque e eixo central e quanto à presença e ausência de tricomas no folíolo. Arachis praecox, representada por um único acesso, aproximou-se mais de $A$. decora que das demais espécies.

Palavras-chave: amendoim-silvestre, caracterização morfológica, Arachis.
\end{abstract}

\section{ABSTRACT \\ MORPHOLOGICAL CHARACTERIZATION OF GERMPLASM ACESSIONS OF FOUR BRAZILIAN SPECIES OF WILD PEANUT}

In this work, a morphological characterization of germplasm accessions of wild Brazilian species of peanut, section Arachis was accomplished. Also, an analysis of the genetic similarity among accessions and between species was evaluated. The experiment was undertaken from 1993 to 1996, at the Campinas Experimental Station of the Instituto Agronômico (IAC). Accessions evaluated were made available by the Wild Arachis Genebank of Embrapa Recursos Genéticos e Biotecnologia (CENARGEN - Brasília, DF) and include A. palustris Krapov., W.C. Gregory \& Valls, A. decora Krapov.,

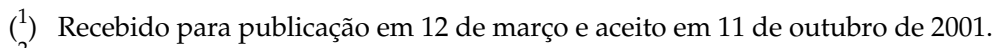

$\left({ }^{2}\right)$ Centro de Recursos Genéticos Vegetais e Jardim Botânico, Instituto Agronômico (IAC), Caixa Postal 28, 13001-970 Campinas (SP). E-mail: veiga@iac.br

(3) Embrapa Recursos Genéticos e Biotecnologia (CENARGEN), Caixa Postal 02372, 70770-500 Brasília (DF).

$\left({ }^{4}\right)$ Doutoranda com bolsa da FAPESP. Escola Superior de Agricultura "Luiz de Queiroz", Universidade de São Paulo, Caixa Postal 83, 13418-900 Piracicaba (SP).

$\left({ }^{5}\right)$ Com bolsa de produtividade em pesquisa do CNPq. 
W.C. Gregory \& Valls, A. praecox Krapov., W.C. Gregory \& Valls and A. stenosperma Krapov. \& W.C. Gregory. Quantitative and qualitative phenotypic data were collected according a list of morphological descriptors. Results showed that accessions of A. stenosperma are very similar, despite the large geographical distance between them. Also, this species forms an isolated group, distant from the other species. Characters such as main stem diameter, fruit and seeds length were useful to separate $A$. stenosperma from the other species. Arachis decora has a high variation between accessions for many characters used. Arachis palustris shows high morphological variation between accessions, although only two accessions were studied, concerning plant height, seed width, peg, isthmus, leaflet, rachis, and main stem dimensions, presence or absence of hairs on the leaflet. Arachis praecox, represented in this work by a single accession, is more similar to $A$. decora than to other species.

Key words: wild peanut, morphological characterization, Arachis.

\section{INTRODUÇÃO}

O gênero Arachis L. (Leguminosae) é dividido em nove seções. Destas, a Arachis mostra maior interesse econômico por abrigar o amendoim comum (Arachis hypogaea L.). Atualmente, essa seção é composta por 27 espécies e, segundo VALLS e SIMPSON (1997), há mais quatro espécies a serem descritas em seu âmbito.

As espécies A. palustris Krapov., W.C. Gregory \& Valls, A. decora Krapov., W.C. Gregory \& Valls e A. praecox Krapov., W.C. Gregory \& Valls compõem um grupo que se destaca pela presença de $2 n=18$ cromossomos, enquanto as outras do gênero todo possuem $2 n=20$ ou $2 n=40$ (Fernandez e Krapovickas, 1994; Lavia, 1996, 1998; PeÑaloza e Valls, 1997). A fórmula cariotípica, comprimento cromossômico, tipo de cromossomo SAT, índices centromérico e de assimetria intracromossômica e razão entre braços também são similares entre elas (LAVIA, 1998), assim como é provável sua origem a partir de um ancestral comum (SouZA, 1997).

Além do estudo da variação interespecífica, são muito importantes as comparações intraespecíficas, tais como a de acessos de germoplasma de $A$. stenosperma Krapov. \& W.C. Gregory, cujas origens geográficas são distintas: uma do litoral Atlântico (para onde foi, provavelmente, levada pelo homem) e, outra, do interior de Mato Grosso (aí incluindo acessos ruderais talvez remanescentes de cultivos indígenas, bem como acessos de ocorrência aparentemente natural). Ademais, essa espécie se posiciona próxima às outras três escolhidas para este trabalho (A. palustris, A decora $e$ A. praecox) na chave taxonômica do gênero.

Segundo KrapovicKas e Gregory (1994), é evidente a necessidade de novas pesquisas para o ordenamento específico dentro da seção Arachis. Assim, caracterizaram-se morfologicamente as quatro espécies brasileiras em questão, incluindo os acessos de procedências geográficas distintas, a fim de verificar a variação existente entre eles e entre as espécies da seção Arachis. O conhecimento da variação fenotípica desses acessos poderá ser útil aos taxonomistas de espécies de Arachis e aos melhoristas de amendoim.

\section{MATERIAL E MÉTODOS}

Avaliaram-se 16 acessos de quatro espécies endêmicas do Brasil e situadas por KRAPOVICKAS e GREGORY (1994) na seção Arachis. Os acessos foram disponibilizados pelo Banco Ativo de Germoplasma de Espécies Silvestres de Arachis, da Embrapa Recursos Genéticos e Biotecnologia (CENARGEN - Brasília, DF). Nove pertencem a $A$. decora, dois a $A$. palustris, um a $A$. praecox e quatro a $A$. stenosperma. Os locais de coleta, códigos de acesso, nomes e números dos coletores e coordenadas geográficas são apresentados no quadro 1. Todos os acessos de A. decora foram coletados em Goiás, em áreas próximas, o mesmo ocorrendo para os dois acessos de A. palustris, coletados no Estado do Tocantins. Já para as acessos de A. stenosperma, utilizaram-se dois de Mato Grosso e outros dois do litoral de São Paulo e do Paraná, originários de ambientes ecogeográficos e altitudes distintas. Arachis praecox foi representada por uma única coleta em Mato Grosso.

Os acessos foram semeados anualmente em telado e, depois, transferidos para o campo em parcelas ao acaso, formando canteiros com 1,5 m entre as plantas e $2 \mathrm{~m}$ entre as linhas, no Núcleo Experimental de Campinas, do Instituto Agronômico (IAC), Campinas (SP), Brasil. Analisaram-se cinco plantas de cada acesso em cada ano agrícola, de 1993 a 1996, seguindo a lista de descritores proposta por VeIGA et al. (1986, 1999). Os caracteres vegetativos analisados foram: altura e diâmetro da planta; comprimento e largura dos folíolos; comprimento e largura das partes adnata e livre das estípulas; comprimento e diâmetro da raque, pecíolo, peciólulo e pulvino. As medidas do folíolo foram obtidas dos folíolos das folhas do eixo central, utilizando-se o folíolo superior direito das folhas. Dos caracteres reprodutivos, analisaram-se o comprimento e a altura da quilha, as asas e o estandarte, e o comprimento e diâmetro do tubo do cálice. Do esporão, foram mensurados o comprimento, a inclinação e o comprimento do istmo. Dos segmentos de frutos e das sementes, anotaram-se o comprimento e a largura (Quadros 2 a 9). 
Quadro 1. Dados de passaporte de 16 acessos de quatro espécies brasileiras de amendoim-silvestre da seção Arachis, com o nome científico das espécies, as siglas dos coletores, o município e a localização geográfica

\begin{tabular}{|c|c|c|c|c|c|c|}
\hline Espécies/BRA & Coletores $^{(1)}$ & Município & Estado $^{(2)}$ & Lat. (S) & Long. (W) & Altitude \\
\hline \multirow{2}{*}{\multicolumn{7}{|c|}{ A. decora }} \\
\hline & & & & & & \\
\hline 032069 & WPn 164 & Paranã & $\mathrm{TO}$ & $12^{\circ} 37^{\prime}$ & $47^{\circ} 52^{\prime}$ & 360 \\
\hline 032093 & WPn 171 & Paranã & TO & $12^{\circ} 37^{\prime}$ & $47^{\circ} 52^{\prime}$ & 360 \\
\hline 031241 & WWs 113 & Nova Roma & GO & $13^{\circ} 38^{\prime}$ & $46^{\circ} 50^{\prime}$ & 500 \\
\hline 028681 & SkW 644 & Nova Roma & GO & $13^{\circ} 51^{\prime}$ & $46^{\circ} 52^{\prime}$ & 490 \\
\hline 022985 & VSW 9953 & Campos Belos & GO & $13^{\circ} 01^{\prime}$ & $46^{\circ} 42^{\prime}$ & 690 \\
\hline 022811 (tipo) & VSW 9955 & Campos Belos & GO & $13^{\circ} 01^{\prime}$ & $46^{\circ} 42^{\prime}$ & 690 \\
\hline 029882 & VPmSv 12900 & Terezina & GO & $13^{\circ} 26^{\prime}$ & $47^{\circ} 08^{\prime}$ & 390 \\
\hline 030902 & VSPmPzRsWi 13290 & Monte Alegre & GO & $13^{\circ} 18^{\prime}$ & $46^{\circ} 42^{\prime}$ & 500 \\
\hline 030970 & VSPmPzRsWi 13307 & Iaciara & GO & $14^{\circ} 03^{\prime}$ & $46^{\circ} 50^{\prime}$ & 450 \\
\hline \multicolumn{7}{|l|}{ A. palustris } \\
\hline 014419 (tipo) & VKRSv 6536 & Miracema & TO & $09^{\circ} 29^{\prime}$ & $48^{\circ} 36^{\prime}$ & 250 \\
\hline 030058 & VPmSv 13023 & Filadelfia & $\mathrm{TO}$ & $07^{\circ} 25^{\prime}$ & $47^{\circ} 37^{\prime}$ & 200 \\
\hline \multicolumn{7}{|l|}{ A. praecox } \\
\hline 012126 (tipo) & VSGr 6416 & Cáceres & MT & $15^{\circ} 39^{\prime}$ & $57^{\circ} 15^{\prime}$ & 215 \\
\hline \multicolumn{7}{|l|}{ A. stenosperma } \\
\hline 016055 & VSMGeSv 7377 & Antonina & PR & $25^{\circ} 26^{\prime}$ & $48^{\circ} 43^{\prime}$ & 3 \\
\hline 018091 & VSStGdW 7762 & Araguaiana & MT & $15^{\circ} 32^{\prime}$ & $52^{\circ} 13^{\prime}$ & 350 \\
\hline 023001 & VmiSv 10229 & Cananéia & $\mathrm{SP}$ & $25^{\circ} 01^{\prime}$ & $47^{\circ} 55^{\prime}$ & 10 \\
\hline 024830 & VSv 10309 & Rondonópolis & MT & $16^{\circ} 28^{\prime}$ & $54^{\circ} 39^{\prime}$ & 215 \\
\hline
\end{tabular}

$\left({ }^{1}\right)$ Coletores: Gd = I.J. de Godoy; Ge = M.A.N. Gerin; Gr = A. Gripp; K= A. Krapovickas; M = J.P. Moss; Mi = S.T.S. Miotto; Pm = R.N. Pittman; Pn = P. Pinheiro; Pz = E.A. Pizarro; R = V.R. Rao; Rs = R.C. Santos; S = C.E. Simpson; Sk = L. Skorupa; St = H.T. Stalker; $\mathrm{Sv}=$ G.P. Silva; V = J.F.M. Valls; W = W. Werneck; Wi = D. Williams; Ws = W. Silva.

$\left({ }^{2}\right)$ Estados brasileiros: GO = Goiás; MT = Mato Grosso; PR = Paraná; SP = São Paulo; TO = Tocantins. Obs: As coletas WPn 164 e WPn 171 possuem as mesmas coordenadas, mas situam-se uma em cada lado do rio, próximo à cidade de Paranã (TO).

Estudaram-se os seguintes caracteres qualitativos: cor e densidade dos ramos, cor dos folíolos e das flores, características dos tricomas na face abaxial dos folíolos (presentes ou ausentes, curtos ou longos, densos ou raros), presença ou ausência de marcas lineares no estandarte (visíveis ou não) na face ventral, cor da base do estandarte e das asas, em flores completamente abertas.

Avaliaram-se as médias dos caracteres quantitativos pela análise da variância e do teste de Tukey ao nível de 5\%. Para os caracteres qualitativos, a presença ou a ausência de determinada característica foi considerada para 95\% da amostra. Realizou-se, também, uma análise de agrupamento, utilizando-se o programa NTSYS-pc 2.0 (RoHLF, 1992) e um dendrograma gerado pelo método UPGMA (SNEATH e SOKAL, 1973). Com base nos cálculos, estabeleceu-se um dendrograma, cujas distâncias entre os indivíduos foram convertidas em porcentagens, tomando para esse fim o valor obtido na formação do grupo final igual a $100 \%$. A análise do dendrograma permitiu a verificação do grau de similaridade entre os indivíduos. Estabeleceram-se os grupos de forma subjetiva, tendo por base as mudanças acentuadas de níveis, asso- ciadas ao conhecimento prévio do material avaliado, segundo CRUZ e RegazZI (1997). Calculou-se o coeficiente de correlação $(r)$ pela matriz cofenética a fim de testar a confiabilidade do agrupamento.

De 53 caracteres morfológicos pesquisados - 45 quantitativos e 7 qualitativos -34 foram selecionados para a análise de agrupamento, uma vez que alguns deles não diferenciaram significativamente os acessos, conforme a análise da variância e o teste de Tukey (Quadros 2 a 9). Os 34 caracteres informativos utilizados na análise de agrupamento foram: eixo central: médias de comprimento e diâmetro da raque e pulvino, médias do comprimento do pecíolo e do diâmetro do peciólulo, médias de comprimento e largura do folíolo, da parte adnata da estípula e largura da parte livre da estípula; ramo lateral: médias de comprimento e largura da parte livre da estípula, largura da parte adnata da estípula, médias do diâmetro da raque e do peciólulo; flor: altura da quilha e do estandarte, comprimento do tubo do cálice, diâmetro do cálice, presença de marcas lineares e cor do estandarte; esporão: comprimento e inclinação; fruto: comprimento e largura; semente: comprimento e largura; 
Quadro 2. Médias da altura (A) e do diâmetro (D) da planta e do comprimento (C) e largura (L) dos frutos e sementes de 16 acessos de quatro espécies brasileiras de amendoim-silvestre da seção Arachis, 1993-1996, Campinas (SP)

\begin{tabular}{|c|c|c|c|c|c|c|}
\hline \multirow{2}{*}{ Espécies e acessos } & \multicolumn{2}{|c|}{ Planta } & \multicolumn{2}{|c|}{ Fruto } & \multicolumn{2}{|c|}{ Semente } \\
\hline & A & $\mathrm{D}$ & $\mathrm{C}$ & $\mathrm{L}$ & $\mathrm{C}$ & $\mathrm{L}$ \\
\hline & & & & & & \\
\hline \multicolumn{7}{|l|}{ A. decora } \\
\hline W 113 & $8,20 \mathrm{fgh}$ & $100,80 \mathrm{c}-\mathrm{f}$ & $1,14 \mathrm{~cd}$ & $0,68 \mathrm{ab}$ & $0,97 \mathrm{~b}$ & $0,54 \mathrm{a}$ \\
\hline W 164 & $3,80 \mathrm{~h}$ & $77,60 \mathrm{f}$ & $1,13 \mathrm{~cd}$ & $0,63 \mathrm{ab}$ & $0,93 \mathrm{~b}$ & $0,49 \mathrm{ab}$ \\
\hline W 171 & $7,80 \mathrm{fgh}$ & $106,20 \mathrm{c}-\mathrm{f}$ & $1,14 \mathrm{~cd}$ & $0,59 \mathrm{ab}$ & $0,94 \mathrm{~b}$ & $0,51 \mathrm{ab}$ \\
\hline Sk 644 & $7,80 \mathrm{fgh}$ & 83,80 ef & $1,14 \mathrm{~cd}$ & $0,62 \mathrm{ab}$ & $0,90 \mathrm{~b}$ & $0,49 \mathrm{ab}$ \\
\hline V 9953 & $15,80 \mathrm{bcd}$ & $101,00 \mathrm{c}-\mathrm{f}$ & $1,14 \mathrm{~cd}$ & $0,65 \mathrm{ab}$ & $0,95 \mathrm{~b}$ & $0,50 \mathrm{ab}$ \\
\hline V 9955 & $11,60 \mathrm{def}$ & $75,60 \mathrm{f}$ & $1,06 \mathrm{~cd}$ & $0,62 \mathrm{ab}$ & $0,89 \mathrm{~b}$ & $0,49 \mathrm{ab}$ \\
\hline V 12900 & $14,00 \mathrm{cde}$ & 90,40 ef & $1,19 \mathrm{c}$ & $0,62 \mathrm{ab}$ & $0,98 \mathrm{~b}$ & $0,51 \mathrm{ab}$ \\
\hline V 13290 & $7,80 \mathrm{fgh}$ & 125,20 cde & $1,13 \mathrm{~cd}$ & $0,54 \mathrm{~b}$ & $0,89 \mathrm{~b}$ & $0,48 \mathrm{ab}$ \\
\hline V 13307 & $9,20 \mathrm{efg}$ & $146,60 \mathrm{bc}$ & $1,02 \mathrm{~d}$ & $0,59 \mathrm{ab}$ & $0,87 \mathrm{~b}$ & $0,50 \mathrm{ab}$ \\
\hline \multicolumn{7}{|l|}{ A. palustris } \\
\hline V 6536 & $19,60 \mathrm{ab}$ & $138,40 \mathrm{bcd}$ & $1,08 \mathrm{~cd}$ & $0,57 \mathrm{ab}$ & $0,90 \mathrm{~b}$ & $0,42 \mathrm{~b}$ \\
\hline V 13023 & $11,80 \mathrm{def}$ & $103,00 \mathrm{c}-\mathrm{f}$ & $1,13 \mathrm{~cd}$ & $0,71 \mathrm{a}$ & $0,81 \mathrm{~b}$ & $0,54 \mathrm{a}$ \\
\hline \multicolumn{7}{|l|}{ A. praecox } \\
\hline V 6416 & $5,80 \mathrm{gh}$ & 99,60 def & $1,03 \mathrm{~d}$ & $0,57 \mathrm{ab}$ & $0,85 \mathrm{~b}$ & $0,46 \mathrm{ab}$ \\
\hline \multicolumn{7}{|l|}{ A. stenosperma } \\
\hline V 7377 & 16,80 a-d & $211,40 \mathrm{a}$ & $1,83 \mathrm{a}$ & $0,66 \mathrm{ab}$ & $1,46 \mathrm{a}$ & $0,52 \mathrm{a}$ \\
\hline V 7762 & $19,00 \mathrm{abc}$ & $205,60 \mathrm{a}$ & $1,65 b$ & $0,62 \mathrm{ab}$ & $1,39 \mathrm{a}$ & $0,52 \mathrm{a}$ \\
\hline V 10229 & $21,60 \mathrm{a}$ & $227,80 \mathrm{a}$ & $1,64 \mathrm{~b}$ & $0,65 \mathrm{ab}$ & $1,38 \mathrm{a}$ & $0,50 \mathrm{ab}$ \\
\hline V 10309 & $19,00 \mathrm{abc}$ & $183,40 \mathrm{ab}$ & $1,76 \mathrm{ab}$ & $0,65 \mathrm{ab}$ & $1,38 \mathrm{a}$ & $0,54 \mathrm{a}$ \\
\hline
\end{tabular}

Médias seguidas por letras distintas diferem, entre si, ao nível de 5\% pelo teste de Tukey.

Quadro 3. Médias de comprimento (C) e diâmetro (D) da raque, pecíolo, peciólulo e pulvino obtidas dos folíolos do eixo central de 16 acessos de quatro espécies brasileiras de amendoim-silvestre da seção Arachis, 1993-1996, Campinas (SP)

\begin{tabular}{|c|c|c|c|c|c|c|c|c|}
\hline \multirow{2}{*}{ Espécies e acessos } & \multicolumn{2}{|c|}{ Raque } & \multicolumn{2}{|c|}{ Pecíolo } & \multicolumn{2}{|c|}{ Peciólulo } & \multicolumn{2}{|c|}{ Pulvino } \\
\hline & C & $\mathrm{D}$ & C & $\mathrm{D}$ & C & $\mathrm{D}$ & $\mathrm{C}$ & $\mathrm{D}$ \\
\hline \multicolumn{9}{|l|}{ A. decora } \\
\hline W 113 & 0,48 ef & $0,08 \mathrm{abc}$ & 2,32 cde & $0,09 \mathrm{a}$ & $0,08 \mathrm{a}$ & $0,06 \mathrm{c}$ & 0,17 b-e & 0,11 cde \\
\hline W 164 & $0,43 \mathrm{f}$ & 0,05 def & $1,68 \mathrm{e}$ & $0,06 \mathrm{a}$ & $0,06 \mathrm{a}$ & $0,05 \mathrm{c}$ & $0,11 \mathrm{e}$ & 0,08 ef \\
\hline W 171 & 0,50 ef & $0,08 \mathrm{a}-\mathrm{d}$ & $2,08 \mathrm{e}$ & $0,10 \mathrm{a}$ & $0,09 \mathrm{a}$ & $0,05 \mathrm{~cd}$ & 0,16 cde & 0,11 cde \\
\hline Sk 644 & 0,44 ef & $0,08 \mathrm{abc}$ & 2,58 b-e & $0,10 \mathrm{a}$ & $0,12 \mathrm{a}$ & $0,06 \mathrm{bc}$ & $0,20 \mathrm{bcd}$ & $0,15 \mathrm{abc}$ \\
\hline V 9953 & $0,80 \mathrm{bcd}$ & 0,07 b-e & 2,42 cde & $0,11 \mathrm{a}$ & $0,10 \mathrm{a}$ & $0,05 \mathrm{~cd}$ & 0,17 cde & 0,11 cde \\
\hline V 9955 & 0,50 ef & 0,08 a-e & 2,58 b-e & $0,09 \mathrm{a}$ & $0,10 \mathrm{a}$ & $0,05 \mathrm{~cd}$ & $0,19 \mathrm{bcd}$ & 0,10 def \\
\hline V 12900 & $0,78 \mathrm{bcd}$ & $0,09 \mathrm{abc}$ & 3,22 a-d & $0,12 \mathrm{a}$ & $0,10 \mathrm{a}$ & $0,06 \mathrm{c}$ & $0,25 \mathrm{~b}$ & $0,16 \mathrm{ab}$ \\
\hline V 13290 & 0,57 def & 0,05 ef & 2,30 cde & $0,09 \mathrm{a}$ & $0,09 \mathrm{a}$ & $0,04 \mathrm{~cd}$ & 0,14 de & 0,11 cde \\
\hline V 13307 & 0,67 cde & 0,07 b-e & $3,44 \mathrm{ab}$ & $0,10 \mathrm{a}$ & $0,09 \mathrm{a}$ & $0,08 \mathrm{ab}$ & 0,18 b-e & $0,14 \mathrm{a}-\mathrm{d}$ \\
\hline \multicolumn{9}{|l|}{ A. palustris } \\
\hline V 6536 & 0,52 ef & 0,07 c-f & 2,22 de & $0,08 \mathrm{a}$ & $0,06 \mathrm{a}$ & $0,05 \mathrm{~cd}$ & 0,13 de & 0,10 def \\
\hline V 13023 & $0,80 \mathrm{bcd}$ & $0,09 \mathrm{abc}$ & 2,54 b-e & $0,11 \mathrm{a}$ & $0,10 \mathrm{a}$ & $0,06 \mathrm{c}$ & 0,17 cde & 0,12 b-e \\
\hline \multicolumn{9}{|l|}{ A. praecox } \\
\hline V 6416 & 0,46 ef & $0,10 \mathrm{a}$ & $2,08 \mathrm{e}$ & $0,07 \mathrm{a}$ & $0,04 \mathrm{a}$ & $0,03 \mathrm{~d}$ & $0,11 \mathrm{e}$ & $0,07 \mathrm{f}$ \\
\hline \multicolumn{9}{|l|}{ A. stenosperma } \\
\hline V 7377 & $0,94 \mathrm{ab}$ & $0,09 \mathrm{abc}$ & $3,90 \mathrm{a}$ & $0,13 \mathrm{a}$ & $0,11 \mathrm{a}$ & $0,09 \mathrm{a}$ & $0,37 \mathrm{a}$ & $0,18 \mathrm{a}$ \\
\hline V 7762 & $0,86 \mathrm{bc}$ & $0,10 \mathrm{ab}$ & $3,24 \mathrm{abc}$ & $0,11 \mathrm{a}$ & $0,15 \mathrm{a}$ & $0,05 \mathrm{~cd}$ & $0,19 \mathrm{bcd}$ & 0,12 b-e \\
\hline V 10229 & $1,10 \mathrm{a}$ & $0,10 \mathrm{ab}$ & $3,98 \mathrm{a}$ & $0,14 \mathrm{a}$ & $0,10 \mathrm{a}$ & $0,09 \mathrm{a}$ & $0,23 \mathrm{bc}$ & 0,17 a \\
\hline V 10309 & $0,86 \mathrm{bc}$ & $0,10 \mathrm{ab}$ & $3,46 \mathrm{ab}$ & $0,11 \mathrm{a}$ & $0,05 \mathrm{a}$ & $0,05 \mathrm{~cd}$ & $0,19 \mathrm{bcd}$ & 0,14 a-d \\
\hline
\end{tabular}

Médias seguidas por letras distintas diferem, entre si, ao nível de $5 \%$ pelo teste de Tukey. 
Quadro 4. Médias de comprimento (C) e largura (L) das partes livres e adnatas da estípula e folíolo obtidas dos folíolos do eixo central de 16 acessos de quatro espécies de amendoim-silvestre da seção Arachis, 1993-1996, Campinas (SP)

\begin{tabular}{|c|c|c|c|c|c|c|}
\hline \multirow{3}{*}{ Espécies e acessos } & \multicolumn{4}{|c|}{ Estípula } & \multirow{2}{*}{\multicolumn{2}{|c|}{ Folíolos }} \\
\hline & \multicolumn{2}{|c|}{ Parte adnata } & \multicolumn{2}{|c|}{ Parte livre } & & \\
\hline & $\mathrm{C}$ & $\mathrm{L}$ & C & $\mathrm{L}$ & C & $\mathrm{L}$ \\
\hline & & & 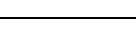 & 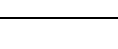 & 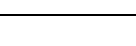 & 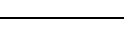 \\
\hline \multicolumn{7}{|l|}{ A. decora } \\
\hline W 113 & $0,82 \mathrm{de}$ & $0,58 \mathrm{ab}$ & 1,80 a-d & $0,21 \mathrm{ab}$ & $2,96 \mathrm{c}-\mathrm{f}$ & $1,20 \mathrm{c}-\mathrm{f}$ \\
\hline W 164 & $0,48 \mathrm{f}$ & $0,41 \mathrm{~d}$ & $0,86 \mathrm{f}$ & $0,11 \mathrm{~b}$ & $1,86 \mathrm{~g}$ & $0,90 \mathrm{f}$ \\
\hline W 171 & 0,72 ef & $0,47 \mathrm{bcd}$ & 0,98 ef & $0,12 \mathrm{ab}$ & $2,52 \mathrm{fg}$ & $1,10 \mathrm{c}-\mathrm{f}$ \\
\hline Sk 644 & $1,00 \mathrm{~b}-\mathrm{e}$ & $0,57 \mathrm{abc}$ & 1,76 a-d & $0,16 \mathrm{ab}$ & 2,82 def & $1,26 \mathrm{~b}-\mathrm{f}$ \\
\hline V 9953 & 0,86 cde & $0,58 \mathrm{ab}$ & $1,96 \mathrm{abc}$ & $0,16 a b$ & $3,28 b-f$ & $1,06 \mathrm{def}$ \\
\hline V 9955 & 0,82 de & $0,52 \mathrm{a}-\mathrm{d}$ & $1,62 \mathrm{~cd}$ & $0,19 a b$ & 2,72 ef & 1,06 def \\
\hline V 12900 & $1,04 \mathrm{bcd}$ & $0,66 \mathrm{a}$ & $1,92 \mathrm{abc}$ & $0,19 a b$ & $3,66 a b c$ & $1,42 \mathrm{a}-\mathrm{d}$ \\
\hline V 13290 & $0,82 \mathrm{de}$ & 0,56 a-d & 1,86 a-d & $0,15 a b$ & 2,72 ef & $1,00 \mathrm{f}$ \\
\hline V 13307 & 0,96 b-e & $0,53 \mathrm{a}-\mathrm{d}$ & $1,66 \mathrm{bcd}$ & $0,19 a b$ & 2,82 def & $1,20 \mathrm{c}-\mathrm{f}$ \\
\hline \multicolumn{7}{|l|}{ A. palustris } \\
\hline V 6536 & $1,10 \mathrm{bcd}$ & $0,64 \mathrm{a}$ & $1,82 \mathrm{a}-\mathrm{d}$ & $0,11 \mathrm{~b}$ & 3,40 a-e & $1,26 b-f$ \\
\hline V 13023 & $1,08 \mathrm{bcd}$ & $0,64 \mathrm{a}$ & $2,18 \mathrm{a}$ & $0,18 a b$ & $3,80 \mathrm{ab}$ & $1,46 a b c$ \\
\hline \multicolumn{7}{|l|}{ A. praecox } \\
\hline V 6416 & 0,98 b-e & $0,52 \mathrm{a}-\mathrm{d}$ & $1,70 \mathrm{bcd}$ & $0,13 a b$ & 2,66 ef & 1,04 ef \\
\hline \multicolumn{7}{|l|}{ A. stenosperma } \\
\hline V 7377 & $1,40 \mathrm{a}$ & $0,60 \mathrm{ab}$ & $2,04 \mathrm{abc}$ & $0,19 a b$ & $3,90 \mathrm{ab}$ & $1,68 \mathrm{a}$ \\
\hline V 7762 & $1,10 \mathrm{bcd}$ & $0,42 \mathrm{~cd}$ & $1,42 \mathrm{de}$ & $0,21 \mathrm{ab}$ & $3,76 \mathrm{ab}$ & 1,38 a-e \\
\hline V 10229 & $1,16 \mathrm{ab}$ & $0,54 \mathrm{a}-\mathrm{d}$ & $2,08 \mathrm{ab}$ & $0,21 a b$ & $4,06 \mathrm{a}$ & $1,62 \mathrm{ab}$ \\
\hline V 10309 & $1,12 \mathrm{abc}$ & $0,54 \mathrm{a}-\mathrm{d}$ & $1,92 \mathrm{abc}$ & $0,22 \mathrm{a}$ & $3,52 \mathrm{a}-\mathrm{d}$ & $1,44 a b c$ \\
\hline
\end{tabular}

Médias seguidas por letras distintas diferem, entre si, ao nível de $5 \%$ pelo teste de Tukey.

Quadro 5. Médias de comprimento (C) e diâmetro (D) da raque, pecíolo, peciólulo e pulvino obtidas dos folíolos do ramo lateral de acessos de quatro espécies brasileiras de amendoim-silvestre da seção Arachis, 1993-1996, Campinas (SP)

\begin{tabular}{|c|c|c|c|c|c|c|c|c|}
\hline \multirow{2}{*}{ Espécies e acessos } & \multicolumn{2}{|c|}{ Raque } & \multicolumn{2}{|c|}{ Pecíolo } & \multicolumn{2}{|c|}{ Peciólulo } & \multicolumn{2}{|c|}{ Pulvino } \\
\hline & $\mathrm{C}$ & $\mathrm{D}$ & $\mathrm{C}$ & $\mathrm{D}$ & $\mathrm{C}$ & $\mathrm{D}$ & $\mathrm{C}$ & $\mathrm{D}$ \\
\hline & & & & & & & & - \\
\hline \multicolumn{9}{|l|}{ A. decora } \\
\hline W 113 & $0,34 \mathrm{a}$ & $0,06 \mathrm{bc}$ & $0,72 \mathrm{a}$ & $0,09 \mathrm{a}$ & $0,10 \mathrm{a}$ & $0,05 \mathrm{abc}$ & $0,13 \mathrm{a}$ & $0,10 \mathrm{a}$ \\
\hline W 164 & $0,31 \mathrm{a}$ & $0,05 \mathrm{bc}$ & $0,72 \mathrm{a}$ & $0,07 \mathrm{a}$ & $0,07 \mathrm{a}$ & $0,04 \mathrm{bc}$ & $0,14 \mathrm{a}$ & $0,08 \mathrm{a}$ \\
\hline W 171 & $0,28 \mathrm{a}$ & $0,05 \mathrm{bc}$ & $0,79 \mathrm{a}$ & $0,06 \mathrm{a}$ & $0,06 \mathrm{a}$ & $0,04 \mathrm{bc}$ & $0,13 \mathrm{a}$ & $0,07 \mathrm{a}$ \\
\hline Sk 644 & $0,33 \mathrm{a}$ & $0,05 \mathrm{bc}$ & $0,44 \mathrm{a}$ & $0,08 \mathrm{a}$ & $0,05 \mathrm{a}$ & $0,04 \mathrm{bc}$ & $0,09 \mathrm{a}$ & $0,09 \mathrm{a}$ \\
\hline V 9953 & $0,31 \mathrm{a}$ & $0,06 \mathrm{abc}$ & $0,76 \mathrm{a}$ & $0,08 \mathrm{a}$ & $0,08 \mathrm{a}$ & $0,03 \mathrm{bc}$ & $0,13 \mathrm{a}$ & $0,09 \mathrm{a}$ \\
\hline V 9955 & $0,34 \mathrm{a}$ & $0,07 \mathrm{ab}$ & $0,76 \mathrm{a}$ & $0,08 \mathrm{a}$ & $0,09 \mathrm{a}$ & $0,05 \mathrm{abc}$ & $0,11 \mathrm{a}$ & $0,09 a$ \\
\hline V 12900 & $0,46 \mathrm{a}$ & $0,06 \mathrm{bc}$ & $0,96 \mathrm{a}$ & $0,10 \mathrm{a}$ & $0,08 \mathrm{a}$ & $0,04 \mathrm{abc}$ & $0,12 \mathrm{a}$ & $0,11 \mathrm{a}$ \\
\hline V 13290 & $0,29 a$ & $0,04 \mathrm{bc}$ & $1,00 \mathrm{a}$ & $0,06 \mathrm{a}$ & $0,05 \mathrm{a}$ & $0,03 \mathrm{c}$ & $0,11 \mathrm{a}$ & $0,09 \mathrm{a}$ \\
\hline V 13307 & $0,33 \mathrm{a}$ & $0,05 \mathrm{bc}$ & $0,72 \mathrm{a}$ & $0,08 \mathrm{a}$ & $0,07 \mathrm{a}$ & $0,04 \mathrm{bc}$ & $0,13 \mathrm{a}$ & $0,09 \mathrm{a}$ \\
\hline \multicolumn{9}{|l|}{ A. palustris } \\
\hline V 6536 & $0,34 \mathrm{a}$ & $0,06 \mathrm{bc}$ & $1,06 \mathrm{a}$ & $0,09 \mathrm{a}$ & $0,10 \mathrm{a}$ & $0,05 \mathrm{abc}$ & $0,12 \mathrm{a}$ & $0,09 \mathrm{a}$ \\
\hline V 13023 & $0,44 \mathrm{a}$ & $0,07 \mathrm{ab}$ & $0,62 \mathrm{a}$ & $0,08 \mathrm{a}$ & $0,08 \mathrm{a}$ & $0,06 \mathrm{a}$ & $0,10 \mathrm{a}$ & $0,09 \mathrm{a}$ \\
\hline \multicolumn{9}{|l|}{ A. praecox } \\
\hline V 6416 & $0,34 \mathrm{a}$ & $0,04 \mathrm{bc}$ & $0,96 \mathrm{a}$ & $0,08 \mathrm{a}$ & $0,05 \mathrm{a}$ & $0,03 \mathrm{bc}$ & $0,09 \mathrm{a}$ & $0,08 \mathrm{a}$ \\
\hline \multicolumn{9}{|l|}{ A. stenosperma } \\
\hline V 7377 & $0,49 \mathrm{a}$ & $0,05 \mathrm{bc}$ & $0,72 \mathrm{a}$ & $0,06 \mathrm{a}$ & $0,09 \mathrm{a}$ & $0,05 \mathrm{abc}$ & $0,15 \mathrm{a}$ & $0,09 \mathrm{a}$ \\
\hline V 7762 & $0,43 a$ & $0,04 \mathrm{c}$ & $0,50 \mathrm{a}$ & $0,09 \mathrm{a}$ & $0,10 \mathrm{a}$ & $0,04 \mathrm{abc}$ & $0,14 \mathrm{a}$ & $0,09 \mathrm{a}$ \\
\hline V 10229 & $0,44 \mathrm{a}$ & $0,06 \mathrm{abc}$ & $0,60 \mathrm{a}$ & $0,10 \mathrm{a}$ & $0,12 \mathrm{a}$ & $0,05 \mathrm{ab}$ & $0,16 \mathrm{a}$ & $0,09 \mathrm{a}$ \\
\hline V 10309 & $0,37 \mathrm{a}$ & $0,09 \mathrm{a}$ & $0,70 \mathrm{a}$ & $0,10 \mathrm{a}$ & $0,09 \mathrm{a}$ & $0,05 \mathrm{abc}$ & $0,13 \mathrm{a}$ & $0,10 \mathrm{a}$ \\
\hline
\end{tabular}

Médias seguidas por letras distintas diferem, entre si, ao nível de $5 \%$ pelo teste de Tukey. 
Quadro 6. Médias de comprimento (C) e largura (L) das partes livres e adnatas da estípula e do folíolo obtidas dos folíolos do ramo lateral de 16 acessos de quatro espécies brasileiras de amendoim-silvestre da seção Arachis, 1993-1996, Campinas (SP)

\begin{tabular}{|c|c|c|c|c|c|c|}
\hline \multirow{3}{*}{ Espécies e acessos } & \multicolumn{4}{|c|}{ Estípula } & \multirow{2}{*}{\multicolumn{2}{|c|}{ Folíolo }} \\
\hline & \multicolumn{2}{|c|}{ Adnata } & \multicolumn{2}{|c|}{ Livre } & & \\
\hline & $\mathrm{C}$ & $\mathrm{L}$ & $\mathrm{C}$ & $\mathrm{L}$ & $\mathrm{C}$ & $\mathrm{L}$ \\
\hline \multicolumn{7}{|l|}{ A. decora } \\
\hline W 113 & $0,48 \mathrm{a}$ & $0,48 \mathrm{abc}$ & $1,14 \mathrm{ab}$ & $0,41 \mathrm{a}$ & $1,68 \mathrm{a}$ & $1,16 \mathrm{ab}$ \\
\hline W 164 & $0,50 \mathrm{a}$ & $0,41 \mathrm{bc}$ & $0,80 \mathrm{~b}$ & $0,10 \mathrm{f}$ & $1,50 \mathrm{a}$ & $0,88 \mathrm{ab}$ \\
\hline W 171 & $0,41 \mathrm{a}$ & $0,37 \mathrm{c}$ & $0,78 \mathrm{~b}$ & $0,18 \mathrm{c}-\mathrm{f}$ & $1,38 \mathrm{a}$ & $0,96 \mathrm{ab}$ \\
\hline Sk 644 & $0,54 \mathrm{a}$ & $0,50 \mathrm{abc}$ & $0,96 \mathrm{ab}$ & $0,17 \mathrm{c}-\mathrm{f}$ & $1,50 \mathrm{a}$ & $1,00 \mathrm{ab}$ \\
\hline V 9953 & $0,48 \mathrm{a}$ & $0,48 \mathrm{abc}$ & $1,12 \mathrm{ab}$ & $0,18 \mathrm{c}-\mathrm{f}$ & $1,50 \mathrm{a}$ & $0,86 \mathrm{ab}$ \\
\hline V 9955 & $0,56 \mathrm{a}$ & $0,46 \mathrm{abc}$ & $0,86 \mathrm{ab}$ & $0,35 \mathrm{ab}$ & $1,54 \mathrm{a}$ & $0,94 \mathrm{ab}$ \\
\hline V 12900 & $0,61 \mathrm{a}$ & $0,52 \mathrm{abc}$ & $1,14 \mathrm{ab}$ & $0,17 \mathrm{c}-\mathrm{f}$ & $1,78 \mathrm{a}$ & $1,00 \mathrm{ab}$ \\
\hline V 13290 & $0,52 \mathrm{a}$ & $0,47 \mathrm{abc}$ & $1,14 \mathrm{ab}$ & $0,16 \mathrm{c}-\mathrm{f}$ & $1,48 \mathrm{a}$ & $0,84 \mathrm{~b}$ \\
\hline V 13307 & $0,48 \mathrm{a}$ & $0,46 \mathrm{abc}$ & $1,16 \mathrm{ab}$ & $0,17 \mathrm{c}-\mathrm{f}$ & $1,48 \mathrm{a}$ & $0,88 \mathrm{ab}$ \\
\hline \multicolumn{7}{|l|}{ A. palustris } \\
\hline V 6536 & $0,52 \mathrm{a}$ & $0,56 \mathrm{ab}$ & $1,16 \mathrm{ab}$ & $0,17 \mathrm{c}-\mathrm{f}$ & $1,66 \mathrm{a}$ & $0,98 \mathrm{ab}$ \\
\hline V 13023 & $0,66 \mathrm{a}$ & $0,58 \mathrm{a}$ & $1,14 \mathrm{ab}$ & 0,15 def & $1,84 \mathrm{a}$ & $1,20 a b$ \\
\hline \multicolumn{7}{|l|}{ A. praecox } \\
\hline V 6416 & $0,47 \mathrm{a}$ & $0,52 \mathrm{abc}$ & $1,31 \mathrm{a}$ & 0,13 ef & $1,70 \mathrm{a}$ & $0,96 \mathrm{ab}$ \\
\hline \multicolumn{7}{|l|}{ A. stenosperma } \\
\hline V 7377 & $0,47 \mathrm{a}$ & $0,40 \mathrm{c}$ & $1,04 \mathrm{ab}$ & $0,42 \mathrm{a}$ & $1,84 \mathrm{a}$ & $1,00 \mathrm{ab}$ \\
\hline V 7762 & $0,52 \mathrm{a}$ & $0,52 \mathrm{abc}$ & $1,02 \mathrm{ab}$ & $0,26 \mathrm{bc}$ & $1,84 \mathrm{a}$ & $1,14 \mathrm{ab}$ \\
\hline V 10229 & $0,38 \mathrm{a}$ & $0,39 c$ & $1,10 \mathrm{ab}$ & 0,22 cde & $1,98 \mathrm{a}$ & $1,22 \mathrm{a}$ \\
\hline V 10309 & $0,54 \mathrm{a}$ & $0,50 \mathrm{abc}$ & $0,94 \mathrm{ab}$ & $0,25 \mathrm{bcd}$ & $1,58 \mathrm{a}$ & $1,04 \mathrm{ab}$ \\
\hline
\end{tabular}

Médias seguidas por letras distintas diferem, entre si, ao nível de $5 \%$ pelo teste de Tukey.

Quadro 7. Médias de comprimento (C) e altura (A) da quilha, asas e estandarte e do comprimento e diâmetro do tubo do cálice de flores de 16 acessos de quatro espécies brasileiras de amendoim-silvestre da seção Arachis, 1993-1996, Campinas (SP)

\begin{tabular}{|c|c|c|c|c|c|c|c|c|}
\hline \multirow{2}{*}{ Espécies e acessos } & \multicolumn{2}{|c|}{ Quilha } & \multicolumn{2}{|c|}{ Asas } & \multicolumn{2}{|c|}{ Estandarte } & \multicolumn{2}{|c|}{ Tubo do cálice } \\
\hline & C & A & C & A & $\mathrm{C}$ & A & $\mathrm{C}$ & D \\
\hline \multicolumn{9}{|l|}{ A. decora } \\
\hline W 113 & $0,10 \mathrm{a}$ & $0,67 \mathrm{c}$ & 0,36 b-e & 0,60 cde & $1,08 \mathrm{bcd}$ & $0,54 \mathrm{de}$ & 2,14 def & $0,06 \mathrm{~cd}$ \\
\hline W 164 & $0,10 \mathrm{a}$ & $0,60 \mathrm{c}$ & 0,29 def & 0,52 cde & 0,82 def & 0,58 cde & $2,30 \mathrm{c}-\mathrm{f}$ & $0,06 \mathrm{~cd}$ \\
\hline W 171 & $0,10 \mathrm{a}$ & $0,60 \mathrm{c}$ & 0,34 b-f & 0,52 cde & 0,68 ef & $0,81 \mathrm{bc}$ & 1,82 ef & $0,06 \mathrm{~cd}$ \\
\hline Sk 644 & $0,14 \mathrm{a}$ & $0,64 \mathrm{c}$ & $0,31 \mathrm{c}-\mathrm{f}$ & 0,56 cde & 0,96 cde & $0,71 \mathrm{~cd}$ & 2,96 b-e & $0,06 \mathrm{~cd}$ \\
\hline V 9953 & $0,10 \mathrm{a}$ & $0,70 \mathrm{bc}$ & 0,29 def & 0,58 cde & 0,94 cde & 0,60 cde & $3,62 \mathrm{a}-\mathrm{d}$ & $0,07 \mathrm{bcd}$ \\
\hline V 9955 & $0,10 \mathrm{a}$ & $0,64 \mathrm{c}$ & 0,34 b-f & 0,56 cde & 0,72 ef & $0,44 \mathrm{e}$ & 1,39 ef & $0,06 \mathrm{~cd}$ \\
\hline V 12900 & $0,10 \mathrm{a}$ & $0,59 \mathrm{c}$ & 0,22 ef & $0,50 \mathrm{de}$ & 0,68 ef & $0,43 \mathrm{e}$ & 1,36 ef & $0,05 \mathrm{~d}$ \\
\hline V 13290 & $0,10 \mathrm{a}$ & $0,62 \mathrm{c}$ & 0,29 def & 0,52 cde & 0,88 cde & 0,54 de & 2,44 cde & $0,06 \mathrm{bcd}$ \\
\hline V 13307 & $0,10 \mathrm{a}$ & $0,68 \mathrm{c}$ & $0,37 \mathrm{bcd}$ & $0,69 \mathrm{bc}$ & $1,18 \mathrm{bc}$ & $0,73 \mathrm{~cd}$ & $3,50 \mathrm{a}-\mathrm{d}$ & $0,08 \mathrm{abc}$ \\
\hline \multicolumn{9}{|l|}{ A. palustris } \\
\hline V 6536 & $0,10 \mathrm{a}$ & $0,67 \mathrm{c}$ & $0,31 \mathrm{c}-\mathrm{f}$ & 0,60 cde & 0,88 cde & 0,52 de & 2,83 b-e & $0,06 \mathrm{~cd}$ \\
\hline V 13023 & $0,10 \mathrm{a}$ & $0,60 \mathrm{c}$ & $0,37 \mathrm{bcd}$ & $0,68 \mathrm{bcd}$ & $1,08 \mathrm{bcd}$ & 0,66 cde & $2,38 c-f$ & $0,06 \mathrm{~cd}$ \\
\hline \multicolumn{9}{|l|}{ A. praecox } \\
\hline V 6416 & $0,10 \mathrm{a}$ & $0,66 \mathrm{c}$ & $0,21 \mathrm{f}$ & $0,48 \mathrm{e}$ & $0,50 \mathrm{f}$ & $0,42 \mathrm{e}$ & $0,82 \mathrm{f}$ & $0,05 \mathrm{~d}$ \\
\hline \multicolumn{9}{|l|}{ A. stenosperma } \\
\hline V 7377 & $0,10 \mathrm{a}$ & 0,93 a & $0,52 \mathrm{a}$ & $0,94 \mathrm{a}$ & $1,66 \mathrm{a}$ & $1,10 \mathrm{a}$ & $4,38 \mathrm{ab}$ & $0,10 \mathrm{a}$ \\
\hline V 7762 & $0,10 \mathrm{a}$ & 0,91 a & 0,40 a-d & $0,86 \mathrm{ab}$ & $1,16 \mathrm{bcd}$ & $1,02 \mathrm{ab}$ & $4,64 \mathrm{a}$ & 0,10 a \\
\hline V 10229 & $0,10 \mathrm{a}$ & $0,87 \mathrm{a}$ & $0,46 \mathrm{ab}$ & $0,94 \mathrm{a}$ & $1,38 \mathrm{ab}$ & $1,08 \mathrm{a}$ & 3,74 a-d & $0,10 \mathrm{a}$ \\
\hline V 10309 & $0,10 \mathrm{a}$ & $0,85 \mathrm{ab}$ & $0,45 \mathrm{abc}$ & $0,79 \mathrm{ab}$ & $1,40 \mathrm{ab}$ & $1,00 \mathrm{ab}$ & $3,80 \mathrm{abc}$ & $0,09 \mathrm{ab}$ \\
\hline
\end{tabular}

Médias seguidas por letras distintas diferem, entre si, ao nível de $5 \%$ pelo teste de Tukey. 
Quadro 8. Médias do comprimento (C) e da inclinação (I) do esporão e do comprimento (c) do istmo de flores de 16 acessos de quatro espécies brasileiras de amendoim-silvestre da seção Arachis, 1993-1996, Campinas (SP)

\begin{tabular}{lrrc}
\hline \multirow{4}{*}{ Espécies e acessos } & \multicolumn{2}{c}{ Esporão } & \multirow{2}{*}{ Istmo } \\
\cline { 2 - 3 } & $\mathrm{C}$ & $\mathrm{I}$ & $\mathrm{C}$ \\
\cline { 2 - 3 } A. decora & & & \\
W 113 & $9,00 \mathrm{~h}$ & $50,00 \mathrm{~b}$ & $0,70 \mathrm{e}$ \\
W 164 & $6,52 \mathrm{k}$ & $35,00 \mathrm{~d}$ & $0,60 \mathrm{f}$ \\
W 171 & $9,00 \mathrm{~h}$ & $40,00 \mathrm{c}$ & $0,70 \mathrm{e}$ \\
Sk 644 & $10,00 \mathrm{~g}$ & $40,00 \mathrm{c}$ & $0,70 \mathrm{e}$ \\
V 9953 & $6,00 \mathrm{i}$ & $40,00 \mathrm{c}$ & $1,20 \mathrm{~d}$ \\
V 9955 & $9,00 \mathrm{~h}$ & $40,00 \mathrm{c}$ & $0,70 \mathrm{e}$ \\
V 12900 & $10,00 \mathrm{~g}$ & $50,00 \mathrm{~b}$ & $0,70 \mathrm{e}$ \\
V 13290 & $8,00 \mathrm{i}$ & $50,00 \mathrm{~b}$ & $2,00 \mathrm{~b}$ \\
V 13307 & $7,00 \mathrm{j}$ & $40,00 \mathrm{c}$ & $2,00 \mathrm{~b}$ \\
A. palustris & & & \\
V 6536 & $10,50 \mathrm{f}$ & $50,00 \mathrm{~b}$ & $0,60 \mathrm{f}$ \\
V 13023 & $11,50 \mathrm{e}$ & $30,00 \mathrm{e}$ & $1,50 \mathrm{c}$ \\
A. praecox & & & \\
V 6416 & $8,00 \mathrm{i}$ & $40,00 \mathrm{c}$ & $0,50 \mathrm{~g}$ \\
A. stenosperma & & & \\
V 7377 & $18,00 \mathrm{a}$ & $60,00 \mathrm{a}$ & $2,50 \mathrm{a}$ \\
V 7762 & $14,00 \mathrm{bc}$ & $50,00 \mathrm{~b}$ & $2,50 \mathrm{a}$ \\
V 10229 & $15,00 \mathrm{~b}$ & $40,00 \mathrm{c}$ & $2,50 \mathrm{a}$ \\
V 10309 & $13,50 \mathrm{~d}$ & $50,00 \mathrm{~b}$ & $2,50 \mathrm{a}$ \\
\hline
\end{tabular}

Médias seguidas por letras distintas diferem, entre si, ao nível de $5 \%$ pelo teste de Tukey.

folíolos: cor e presença ou ausência de tricomas; planta: altura e diâmetro, cor e densidade dos ramos.

Os dados quantitativos foram transformados em matriz de dados binários (0 e 1), cuja média foi estabelecida como limite para a discriminação. Considerou-se 0 ou 1 para os valores abaixo ou acima da média respectivamente. Mesmo que este artifício tenha causado perda de sensibilidade dos valores (BASSAB et al., 1990), foi a melhor maneira de colocar os dados qualitativos e quantitativos em uma mesma matriz.

Utilizou-se o coeficiente de similaridade de Simple Matching $\left(\mathrm{S}_{\mathrm{sm}}\right)$ para o cálculo da semelhança entre os acessos de uma mesma espécie; o método de agrupamento utilizado foi o UPGMA (SNEATH e SOKAL, 1973).

A matriz de dados binários foi submetida à análise por coordenadas principais (PCO), aplicando-se o programa NTSYS 2.0-pc. Essa análise, que aceita dados binários, pode ser utilizada como alternativa computacional da análise por componentes principais (PCA) (RoHLF, 1992), pois aceita valores com intervalos (quantitativos) e não binários.

\section{RESULTADOS E DISCUSSÃO}

Observa-se pelo quadro 1 que os acessos de $A$. decora foram coletados nos Estados de Goiás e do Tocantins, em áreas relativamente próximas entre si. Já os acessos de $A$. palustris foram coletados no Tocantins, em áreas proporcionalmente distantes. Arachis praecox foi representada por um acesso disponível, a única população conhecida da espécie, localizada em Mato Grosso. Dois acessos de A. stenosperma foram coletados em Mato Grosso e, outros dois, no litoral Atlântico, em São Paulo e no Paraná, portanto, em áreas muito disjuntas. Além das populações serem distantes, a altitude e o ambiente ecogeográfico no Brasil-Central ou no litoral também eram muito diferentes.

Quanto à altura da planta, verifica-se que quase todos os acessos de $A$. decora mostraram tendência de apresentar porte baixo $(<10 \mathrm{~cm})$ juntamente com $A$. praecox (Quadro 2). Já os acessos de A. decora 9953, 12900, 9955 e A. palustris 13023 apresentaram porte intermediário (entre 11 e $16 \mathrm{~cm}$ ). Todos os acessos de A. stenosperma e o acesso 6536 de $A$. palustris apresentaram porte mais alto $(>16 \mathrm{~cm})$.

Os dois acessos de $A$. palustris se diferenciaram pela altura da planta. Enquanto os acessos de $A$. stenosperma e $A$. praecox situaram-se nos pontos extremos, os acessos de $A$. decora mostraram ampla dispersão para esse caráter. Quanto ao diâmetro do eixo central, A. stenosperma apresentou-se significativamente maior que o das outras espécies, sendo o de A. palustris intermediário e o de $A$. praecox, pequeno. Também para esse caráter, $A$. decora apresentou grande variação entre os acessos. $\mathrm{O}$ comprimento dos frutos e das sementes de $A$. stenosperma foi maior que o obtido para as demais espécies; diferenças entre suas larguras não foram perceptíveis.

Nas folhas do eixo central, observaram-se diferenças entre os acessos no comprimento da raque, pecíolo, pulvino, parte livre e adnata da estípula e do folíolo, enquanto o diâmetro variou na raque, peciólulo e pulvino. As diferenças na largura da estípula entre os acessos foram maiores na região adnata, sendo observadas, também, diferenças na largura do folíolo (Quadros 3 e 4). Entretanto, ao verificar os folíolos do ramo lateral, as variações entre os acessos foram menores, diferenciando de alguns acessos apenas nos caracteres diâmetro da raque e do peciólulo, comprimento da parte livre da estípula e largura do folíolo e das partes livre e adnata (Quadros 5 e 6).

Quanto à corola (Quadro 7), observou-se que $A$. stenosperma se diferenciava das demais pela altura da quilha, enquanto seu comprimento era igual entre todas as espécies. Encontrou-se grande semelhança 
Quadro 9. Caracteres qualitativos avaliados: cor (cr) e densidade (d) dos ramos; cor dos folíolos (cf) e características dos tricomas da face abaxial dos folíolos; marcas lineares no estandarte (ml), cor da base do estandarte (ce) e cor das asas (ca), obtidos dos 16 acessos de quatro espécies brasileiras de amendoim-silvestre da seção Arachis, 1993-1996, Campinas (SP)

\begin{tabular}{|c|c|c|c|c|c|c|c|}
\hline \multirow{3}{*}{ Espécies e acessos } & \multirow{2}{*}{\multicolumn{2}{|c|}{ Ramos }} & \multirow{2}{*}{\multicolumn{2}{|c|}{ Folíolos }} & \multicolumn{3}{|c|}{ Coloração das flores } \\
\hline & & & & & \multicolumn{2}{|c|}{ Estandarte } & \multirow{2}{*}{$\begin{array}{c}\text { Asas } \\
\text { ca }\end{array}$} \\
\hline & $\mathrm{cr}$ & $\mathrm{d}$ & $\mathrm{cf}$ & (tricomas) & $\mathrm{ml}$ & ce & \\
\hline \multicolumn{8}{|l|}{ A. decora } \\
\hline W 113 & 4 & Raro & 6 & Curtos e raros & Visíveis & 1 & 1 \\
\hline W 164 & 5 & Denso & 5 & Curtos e raros & Visíveis & 2 & 1 \\
\hline W 171 & 4 & Raro & 5 & Curtos e raros & Visíveis & 2 & 1 \\
\hline Sk 644 & 4 & Raro & 5 & Curtos e raros & Visíveis & 1 & 1 \\
\hline V 9953 & 4 & Raro & 6 & Curtos e raros & Visíveis & 2 & 1 \\
\hline V 9955 & 4 & Denso & 6 & Glabros & Visíveis & 2 & 1 \\
\hline V 12900 & 4 & Denso & 5 & Glabros & Visíveis & 2 & 1 \\
\hline V 13290 & 4 & Denso & 5 & Glabros & Visíveis & 2 & 1 \\
\hline V 13307 & 4 & Denso & 6 & Glabros & Visíveis & 2 & 1 \\
\hline \multicolumn{8}{|l|}{ A. palustris } \\
\hline V 6536 & 4 & Raro & 5 & Curtos e densos & Visíveis & 2 & 1 \\
\hline V 13023 & 4 & Raro & 5 & Glabros & Visíveis & 2 & 1 \\
\hline \multicolumn{8}{|l|}{ A. praecox } \\
\hline V 6416 & $4-5$ & Raro & 5 & Curtos e densos & Visíveis & 2 & 1 \\
\hline \multicolumn{8}{|l|}{ A. stenosperma } \\
\hline V 7377 & 4 & Denso & 5 & Curtos e raros & Pouco visíveis & 1 & 1 \\
\hline V 7762 & 4 & Denso & 5 & Curtos e raros & Pouco visíveis & 1 & 1 \\
\hline V 10229 & 4 & Denso & 5 & Curtos e raros & Pouco visíveis & 1 & 1 \\
\hline V 10309 & 4 & Denso & 5 & Curtos e densos & Pouco visíveis & 1 & 1 \\
\hline
\end{tabular}

1 = amarelo; 2 = alaranjado; 3 = arroxeado; 4 = avermelhado; 5 = esverdeado; 6 = verde-escuro. Duas notas representam variação dentro da população.

nas dimensões das asas entre as diferentes espécies e os acessos. Arachis stenosperma também apresentou valores maiores para altura das asas e do estandarte, em relação às demais espécies.

Verifica-se no quadro 8 que $A$. stenosperma possui o comprimento do esporão e do istmo maior que nas demais espécies, variando entre os acessos dessa espécie quanto ao comprimento do esporão.

Quanto aos caracteres morfológicos qualitativos (Quadro 9), observaram-se em A. stenosperma poucas marcas lineares no estandarte, em comparação às demais espécies. Em $A$. decora encontraram-se diferenças entre os acessos nos caracteres cor dos ramos, folíolos e estandarte, bem como na densidade dos ramos e presença ou ausência de tricomas, o que não ocorreu entre os acessos das demais espécies, com exceção da presença ou ausência dos tricomas que também variaram em $A$. palustris e $A$. stenosperma.

Com base nos cálculos, estabeleceu-se um dendrograma, no qual se observa a divisão dos acessos em três grupos distintos (I, II, III) (Figura 1). O grupo I apresentou similaridade genética de 0,32 , em relação aos grupos II e III que englobaram todos os acessos de A. stenosperma. O grupo I pode ser subdividido em 2 subgrupos com os acessos 7377 e 10229, originários do litoral do Paraná e de São Paulo e 7762 e 10309, de Mato Grosso. Nos acessos do grupo I, separados por região geográfica, é possível que um processo incipiente de deriva genética esteja ocorrendo entre os acessos de Mato Grosso e do litoral, uma vez que são submetidos ao isolamento reprodutivo, sofrendo, conseqüentemente, distintas pressões de seleção.

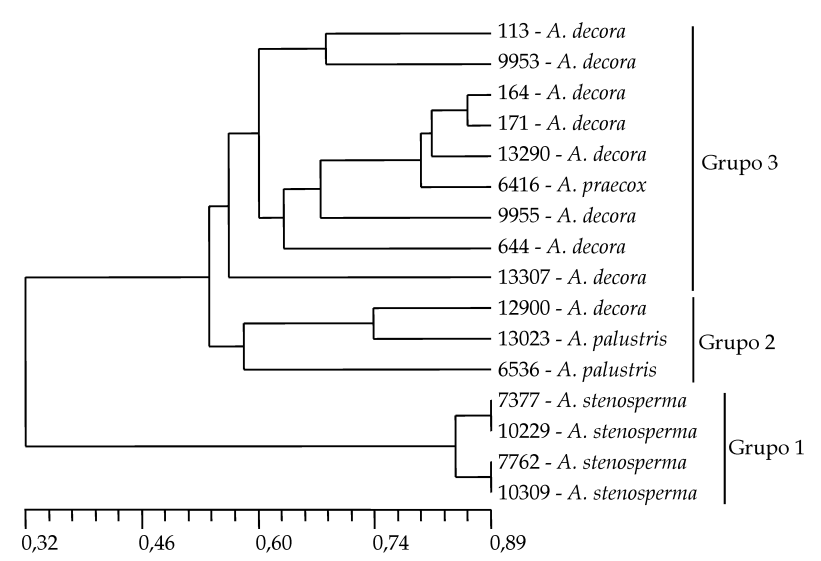

Figura 1. Dendrograma (UPGMA) de 16 acessos de espécies brasileiras da seção Arachis, obtido de 34 caracteres morfológicos, utilizando o coeficiente de Simple Matching. 
O grupo II incluiu os acessos 12900, 13023 e 6536, sendo o primeiro caracterizado taxonomicamente como $A$. decora e os dois últimos, como A. palustris. $\mathrm{O}$ grupo III englobou os acessos de $A$. decora $(113,164$, $171,644,9953,9955,13290$ e 13307) e o acesso de $A$. praecox (6416), mantendo-se isolado do grupo II com similaridade genética de 0,56.

Evidenciou-se, neste trabalho, a distinção de $A$. stenosperma das demais espécies e a similaridade entre os acessos de A. decora, A. praecox e A. palustris, o que confirmou os dados obtidos em caracterização molecular por SouzA (1997). Sabe-se que A. stenosperma é de ciclo perene e possui 20 cromossomos, incluindo um par denominado par "A" (Fernandez e KRAPOVICKAS, 1994; LAVIA, 1996) diferenciado dos demais da mesma célula por sua condensação na prometáfase e por ter a metade, em comprimento, do maior cromossomo na mesma célula. Já as demais espécies são anuais e mostram 18 cromossomos, sem o par " $\mathrm{A}$ " (Lavia, 1996, 1998; PeÑaloza e Valls, 1997). Logo, os agrupamentos observados no dendrograma confirmam tais estudos citogenéticos e morfológicos, estabelecendo nítida diferenciação entre $A$. stenosperma e as outras espécies originalmente descritas como parte da mesma seção Arachis (KRAPOVICKAS e GREGORY, 1994).

A alta similaridade encontrada entre os acessos de A. stenosperma de Mato Grosso e os do litoral Atlântico coincide, em parte, com a forte associação constatada entre tais acessos por MonçATO e VAlLS (1999). Esses autores, entretanto, compararam um número substancialmente maior de diferentes acessos das duas áreas e utilizaram descritores adicionais, como o número de folíolos por folha. Os acessos do litoral mostram, com freqüência, um folíolo adicional, em algumas folhas, até agora não observado em qualquer acesso de Mato Grosso. À exceção desse caráter, a similaridade entre acessos do litoral e de Mato Grosso parece indicar disjunção recente, ou eventual contato ainda existente entre os acessos, o que, no entanto, não pode ser confirmado pelas intensas coletas realizadas.

Quanto à diferenciação entre as espécies com 18 cromossomos, o dendrograma não mostrou grau de similaridade genética suficientemente grande que permitisse a separação dos acessos respeitando a classificação das espécies. O coeficiente de correlação (r) obtido para o agrupamento foi de 0,91 , pois os agrupamentos cujo coeficiente de correlação se encontravam acima de 0,7 (ou 70\%) foram considerados confiáveis, como citado por RoHLF (1992). Esses dados confirmam a grande coesão entre as três espécies de 18 cromossomos, observada por SouZA (1997) a partir de marcadores RAPD.
Por intermédio da análise por coordenadas principais (PCO) foi possível observar os acessos formando agrupamentos mais de acordo com a classificação taxonômica (Figura 2). Quatro agrupamentos foram obtidos, em consonância com a nomenclatura atual. No entanto, foi observada menor distância genética entre os agrupamentos constituídos pelas espécies com 18 cromossomos. Tais dados sugerem certa proximidade entre as espécies, com suspeita de recente especiação. O isolamento reprodutivo em espécies tão próximas pode não ser completo, sendo, neste caso, necessária a realização de cruzamentos controlados que gerem descendentes férteis entre essas espécies, isoladas entre si nos locais de ocorrência natural. $A$. decora, em contraste com as demais espécies, mostrou grande polimorfismo entre as populações, com exceção para os acessos 164 e 171, o que, em parte, se justifica pelo grande número de acessos estudados.

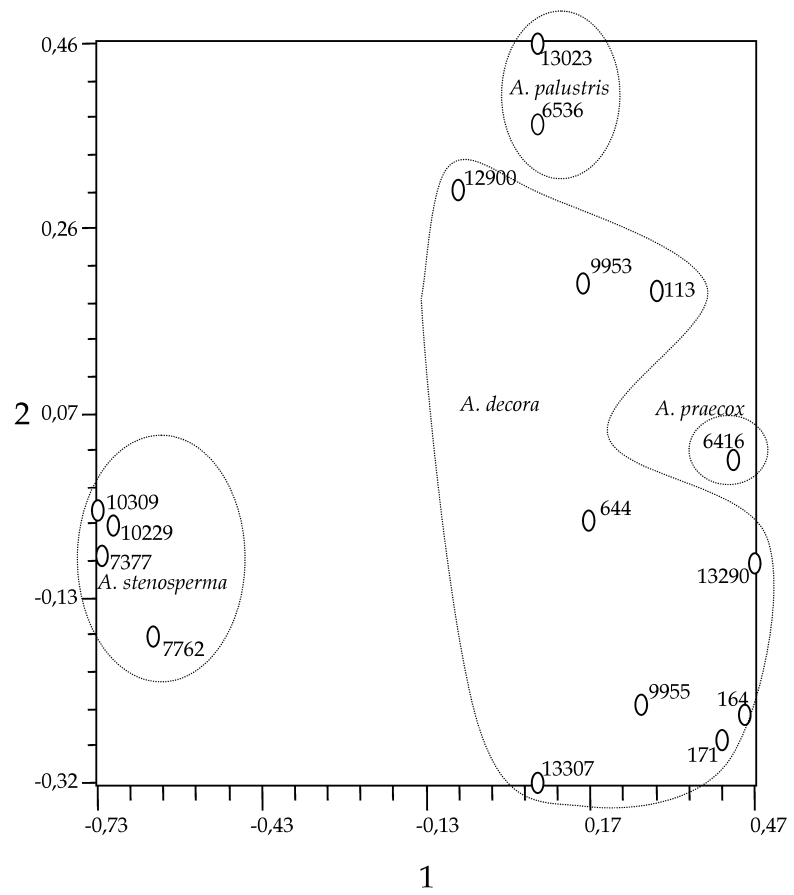

Figura 2. Análise por coordenadas principais (PCO) de 16 acessos de quatro espécies brasileiras da seção Arachis, com base em 34 caracteres morfológicos.

\section{CONCLUSÕES}

1. Das quatro espécies silvestres brasileiras da seção Arachis, analisadas a partir da aplicação de descritores morfológicos, A. stenosperma é a mais distinta, mostrando grande similaridade entre os acessos de áreas geográficas distintas, afastadas mais de $1.000 \mathrm{~km}$, o que sugere tratar-se de disjunção recente.

2. Arachis decora mostra ampla variação morfológica entre os seus acessos, um dos quais aproxima-se 
de A. palustris. Enquanto dois acessos são muito semelhantes entre si, outros são muito distintos, mesmo quando oriundos de coletas próximas.

3. Arachis praecox mostra-se morfologicamente aliada a $A$. decora, situando-se em posição central dentro da variação morfológica apresentada pela primeira espécie. Pelo fato de se apresentarem em áreas geográficas distintas, afastadas mais de $1.000 \mathrm{~km}$, é interessante verificar se há barreiras reprodutivas entre A. praecox e A. decora ou se ocorre disjunção dos acessos de uma mesma espécie, à semelhança do verificado em $A$. stenosperma.

4. Mesmo diferenciando-se de A. decora, A. palustris mostra associação com a primeira espécie, vinculando-se a um de seus acessos. Seria importante verificar a eventual compatibilidade reprodutiva entre $A$. palustris e A. decora.

5. As três espécies diplóides da seção Arachis com 18 cromossomos formam um grupo bastante coeso, do ponto de vista morfológico, e contrastante $\operatorname{com} A$. stenosperma, espécie diplóide com 20 cromossomos. Recomenda-se a procura de novos acessos dessas três espécies nas atuais áreas conhecidas, a fim de documentar eventual ocorrência contínua e não disjunta.

\section{REFERÊNCIAS BIBLIOGRÁFICAS}

BASSAB, W. de O.; MIASAKI, E.S.; ANDRADE, D.F. de. Introdução à análise de agrupamentos. In: SIMPÓSIO NACIONAL DE PROBABILIDADE E ESTATÍSTICA, 9., 1990, São Paulo. Anais... São Paulo: Associação Brasileira de Estatística (ABE), 1990. 105p.

CRUZ, C.D.; REGAZZI, A.J. Modelos biométricos aplicados ao melhoramento genético. Viçosa: UFV, 1997. 390p.

FERNANDEZ, A.; KRAPOVICKAS, A. Cromosomas y evolución en Arachis (Leguminosae). Corrientes, Revista del Instituto de Botánica del Nordeste, Bonplandia, V. 8, n.1/4, p.186-220, 1994.

KRAPOVICKAS, A.; GREGORY W.C. Taxonomía del género Arachis (Leguminosae). Bonplandia, Corrientes, v.8, n.1/4, p.1-186. 1994.

LAVIA, G.I. Estudios cromosómicos en Arachis (Leguminosae). Bonplandia, Corrientes, v.9, p.111-120, 1996.
LAVIA, G.I. Karyotypes of Arachis palustris and A. praecox (Section Arachis), two species with basic chromosome number $\mathrm{x}=$ 9. Cytologia, Tokyo, v.63, p.177-181, 1998.

MONÇATO, L.; VALLS, J.F.M. Similaridad entre Arachis stenosperma y materiales afines del valle del rio Araguaia, Brasil. In: SANCHES, R.; BALDESSARI, J.; GIANDANA, E. (Eds.). ENCUENTRO DE ESPECIALISTAS EN Arachis spp. DE AMERICA LATINA, 2., 1999, Cordoba. Resúmenes... Cordoba: INTA, 1999. p.21.

PEÑALOZA, A.D.P.S.; VALLS, J.F.M. Contagem do número cromossômico em acessos de Arachis decora (Leguminosae). In: VEIGA, R.F.A; BOVI, M.L.A; BETTI, J.A.; VOLTAN, R.B.Q. (Eds.) SIMPÓSIO LATINO-AMERICANO DE RECURSOS GENÉTICOS VEGETAIS, 1., 1997, Campinas. Programas e Resumos... Campinas: IAC/Embrapa-Cenargen, 1997. p.39.

ROHLF, F.J. NTSYS-pc Numerical taxonomy and multivariate analysis system. Version 1.70, New York: Applied Biostatistics, 1992.

SNEATH, P.E.A.; SOKAL, R. Numerical Taxonomy. San Francisco: Freeman, 1973. 573p.

SOUZA, S.A.C.D. de. Caracterização Molecular de Espécies Anuais Brasileiras da Secção Arachis e da Secção Heteranthae, Gênero Arachis. Botucatu, 1997. 56p. Dissertação (Mestrado em Genética) - Universidade Estadual Paulista.

VALLS, J.F.M.; SIMPSON, C.E. Novas espécies de Arachis (Leguminosae). In: VEIGA, R.F.A; BOVI, M.L.A; BETTI, J.A.; QUEIROZ-VOLTAN, R.B. (Eds.). SIMPÓSIO LATINO-AMERICANO DE RECURSOS GENÉTICOS VEGETAIS, 1., 1997, Campinas. Programas e Resumos... Campinas: IAC/Embrapa-Cenargen, 1997. p.27.

VEIGA, R.F.A.; GODOY, I.J.; SAVY-FILHO, A.; GERIN, M.A.N.; VALLS, J.F.M. Descritores de amendoim (Arachis hypogaea L.) utilizados no Instituto Agronômico de Campinas. Campinas: Instituto Agronômico, 1986. 21p. (Boletim Técnico, 28)

VEIGA, R.F.A.; VALLS, J.F.M.; LOPES, C.R.; SIMPSON, C.E. Organographical and agricultural descriptors to Brazilian populations of wild peanut Arachis sylvestris (A.Chev) A.Chev.. Campinas: Instituto Agronômico, 1999. 13p. (Boletim Técnico, 178) 